\title{
The Application and Operation-Effect Analysis for Complex Tibial Plateau Fractures with 3D Printing Technique
}

\author{
Changjin Guo1, Yubo Zhang², Li Yang1, Qiaofeng Zhu' ${ }^{1}$, Sanming Zou² ${ }^{2}$ \\ ${ }^{1}$ Medical College, Wuhan University of Science and Technology, Wuhan, China \\ ${ }^{2}$ Department of Orthopaedics, Xiaogan Central Hospital, Wuhan University of Science and Technology, Xiaogan, China \\ Email: 765312069@qq.com, *xgzsmys@163.com
}

How to cite this paper: Guo, C.J., Zhang, Y.B., Yang, L., Zhu, Q.F. and Zou, S.M. (2019) The Application and OperationEffect Analysis for Complex Tibial Plateau Fractures with 3D Printing Technique. International Journal of Clinical Medicine, 10, 101-108.

https://doi.org/10.4236/ijcm.2019.103010

Received: January 30, 2019

Accepted: March 2, 2019

Published: March 5, 2019

Copyright $\odot 2019$ by author(s) and Scientific Research Publishing Inc. This work is licensed under the Creative Commons Attribution International License (CC BY 4.0).

http://creativecommons.org/licenses/by/4.0/

\begin{abstract}
Objective: To investigate the value of 3D printing techniques in the treatment of complex tibial plateau fractures. Methods: From September 2016 to September 2018, 28 patients with complex tibial plateau fractures were treated in our hospital. According to the odevity of hospitalized order, the patients were divided into two groups. Group A used 3D reconstruction, virtually reduction, 3D printing and demonstration of individual fracture model before operation while group B only received conventional process by use X-rays or CT image. Comparison between the two groups was made in operation time, operative blood loss, radiation frequency, surgery instrument cost and knee function score. Results: The follow-up was 14.4 months on average (ranged 6 to 22 months). There was no statistical difference of the surgery instrument cost between the 2 groups $(P>0.05)$. The operation time of group A was significantly shorter than that of group B (P $<0.05)$. Group A also performed better than group B in comparison of operative blood loss and radiation frequency. The excellent good rate of HSS score in group A was $92.86 \%$; it was higher than that $85.71 \%$ in group B. There was no statistical difference between the 2 groups $\left(\chi^{2}=0.373, \mathrm{P}=\right.$ $0.54)$. Conclusion: $3 \mathrm{D}$ printing techniques can improve surgery effect in complex tibial plateau fractures.
\end{abstract}

\section{Keywords}

Tibial Plateau Fracture, 3D Printing Technique, Individualized Treatment

\section{Introduction}

Complex tibial plateau fracture is one of the most common and complex frac- 
tures in limb trauma, in which the knee joint suffers from inversion or vertical compression violence, resulting in unicondylar or bicondylar fractures of the medial and lateral tibial plateau condyles. Large data analysis shows that tibial plateau fractures account for $1 \%-2 \%$ of the total body fractures and $8 \%$ of the elderly fractures [1]. With the increasing incidence of accidents and traffic accidents in recent years, the incidence of various complex injuries has gradually increased, and the incidence of tibial plateau fractures has also increased. Surgical treatment of complex tibial plateau fractures requires a high standard of surgeons. Since it is a complex intra-articular fracture with high energy, it is often accompanied by severe articular surface collapse, ligament injury around the knee joint and poor soft tissue condition. Therefore, the incidence of early complications such as skin ischemic necrosis, infection, poor wound healing, bone and internal fixation exposure, as well as late complications such as long-term pain, postoperative dysfunction, and traumatic osteoarthritis is higher than other fractures. The way to restore the smooth joint surface as much as possible, and at the same time to achieve the minimum damage to the soft tissue, is the primary problem in front of the surgeon. In recent years, the rapid development of digital software and $3 \mathrm{D}$ printing technique in China has provided a rare opportunity to solve the above problems. Orthopaedics surgeons can design and simulate the operation on the $3 \mathrm{D}$ printing model in advance, so as to improve the surgical treatment effect. In 2014, the team of Professor Wang Liao and Professor Dai Kerong had introduced the individualized orthopaedic treatment and 3D printing technique in detail [2], bringing digital orthopaedic surgery into the vision of domestic orthopaedic physicians. Therefore, the application of 3D printing technique in complex tibial plateau fracture surgery is an effective means to realize the individualization and precision of orthopaedic surgery.

\section{Materials and Methods}

\subsection{General Materials}

28 patients admitted to and treated in our hospital with complex tibial plateau fractures from September 2016 to September 2018 were enrolled in the clinical trial, including 17 males and 11 females, aged from 19 to 62 years, with an average age of 38.8 years. Unified inclusion and exclusion criteria were adopted for the study objects. All patients were with complicated tibial plateau fractures and elective surgery, with informed consent. Patients with pathological fractures, multiple fractures of the same limb, combined with nerve and blood vessel injuries, dislocation of knee joint and intolerance for the surgery were excluded. Preoperative MRI examination was performed to exclude patients with meniscus or ligament injury. According to Schatzker classification: There were 11 cases of type II, 7 cases of type IV, 8 cases of type V and 2 cases of type VI. All the surgeries were performed by the same surgeon and the corresponding team. The patients were divided into groups according to the order of admission. Patients with odd number of admission orders were classified into group $\mathrm{A}$, and patients 
with even number were classified into group B. There were 14 cases in group A, 9 males and 5 females; aged from 19 to 58 years, with an average age of 38.13 years. For the fracture classification: 5 cases of type II, 3 cases of type IV, 5 cases of type V and 1 case of type VI. There were 14 cases in group B, 8 males and 6 females; aged from 21 to 62 years, with an average age of 38.47 years. For the fracture classification: 6 cases of type II, 4 cases of type IV, 3 cases of type V and 1 case of type VI. Inclusion criteria: patients meeting the diagnostic criteria for complex tibial plateau fractures; patients with severe complications and open fractures were excluded. All the patients are able to tolerate surgery and anesthesia, and informed consent to the treatment.

\subsection{Grouping Data Processing and 3D Reconstruction Software Application Analysis}

All patients in group A underwent anteroposterior and lateral films of knee joint, CT plain scan of knee joint and 3D reconstruction before surgery. Professionals imported CT data into Mimics software and convert them into 3D models. The situation of fracture blocks were observed, the pixel set of each bone block was separated to establish a 3D model of each bone block, and each bone block was marked with different colors for fracture classification. All patients in group B underwent anteroposterior and lateral films of knee joint, CT plain scan of knee joint and 3D reconstruction before surgery.

\subsection{Preoperative Virtually Reduction and 3D Printing Model}

For the reconstructed 3D model of bone, according to the actual needs of fracture surgery, the virtually reduction of the main fracture directly related to surgery was completed, and the articular surface of tibial plateau was reconstructed to obtain the $3 \mathrm{D}$ model after reduction. The virtual reduction process was observed to fully understand the size and shape of the fracture block, displacement, reduction mode, movement distance and rotation angle, and determine the individualized surgical plan: the choice of operative approach, the position of the steel plate and the length and direction of the screw. After that, the models of each bone block were integrated and the 1:1 model was printed out hierarchically. After observing the printed fracture model, the surgical team carried out pre-operation process, including understanding the match of the fracture block, simulating the placement of anatomical steel plate, understanding the relationship between the fracture line and the position of the steel plate after reduction, and the design of the position and angle of the cancellous bone screw on the inner and outer platform.

\subsection{Evaluation of efficacy}

Hospital for special surgery (HSS) knee score [3] was used to evaluate the recovery of knee joint function. HSS has a relatively high accuracy in comparing the 
recovery of joint function before and after surgery, especially in the evaluation of early postoperative period, which can comprehensively evaluate the movement of the patellofemoral joint and the femoral condyle.

\subsection{Statistical Method}

Data were analyzed by the statistical software of SPSS 13.0. The operation time and operative blood loss, radiation frequency, surgery instrument cost were expressed by mean (+ standard deviation) $(\mathrm{x} \pm \mathrm{s})$ and $\mathrm{t}$-test was adopted. While the knee function score after operation was tested by $\chi^{2}$ test, the difference was considered statistically significant with $\mathrm{P}<0.05$.

\section{Results}

\subsection{Comparison of Surgical Effects}

There was no significant difference in the surgery instrument cost between group A and group B $(t=1.661, P>0.05)$, indicating that the application of $3 \mathrm{D}$ printing technique had no significant impact on the use of surgery instrument in traditional surgery. While, there were statistically significant differences in operation time, operative blood loss and radiation frequency $(\mathrm{P}<0.05)$, indicating that compared with traditional surgery, the application of $3 \mathrm{D}$ printing technique reduced the operation time and intraoperative fluoroscopy times, thus improving the operation efficiency and safety. See Table 1.

Table 1. Comparison of key intraoperative parameters between group A and group B for complex tibial plateau fractures.

\begin{tabular}{cccccc}
\hline Group & Case & $\begin{array}{c}\text { Operation time } \\
\text { min }\end{array}$ & $\begin{array}{c}\text { Operative } \\
\text { blood loss } \\
\mathrm{ml}\end{array}$ & $\begin{array}{c}\text { Perspective } \\
\text { times } \\
\text { time }\end{array}$ & $\begin{array}{c}\text { Internal fixation } \\
\text { consumables cost } \\
\text { Ten thousand yuan }\end{array}$ \\
\hline Control group & 14 & $122.20 \pm 3.26$ & $447.90 \pm 31.08$ & $4.71 \pm 0.62$ & $3.04 \pm 0.23$ \\
Observation group & 14 & $105.10 \pm 4.14$ & $314.30 \pm 37.04$ & $2.71 \pm 0.40$ & $2.54 \pm 0.18$ \\
$t$ & & $\mathrm{t}=3.237$ & $\mathrm{t}=2.762$ & $\mathrm{t}=2.730$ & $\mathrm{t}=1.661$ \\
$\mathrm{P}$ & $<0.05$ & $<0.05$ & $<0.05$ & $>0.05$ \\
\hline
\end{tabular}

\subsection{Comparison of Functional Recovery after Operation}

Six months after operation, the HSS score was used. In group A, 11 cases were excellent, 2 cases were good, 1 case was fair, the excellent and good rate was 92.86\%. In group B, 5 cases were excellent, 7 cases were good, 2 cases were fair, the excellent and good rate was $85.71 \%$. There was no significant difference between the two groups $\left(\chi^{2}=0.373, \mathrm{P}=0.54\right)$ for the excellent and good rate, while the difference of the excellent rate between the two groups was statistically significant $\left(\chi^{2}=5.250, \mathrm{P}<0.05\right)$, indicating that the application of $3 \mathrm{D}$ printing technique can achieve better long-term postoperative functional recovery and better patient satisfaction. See Table 2, Figure 1 and Figure 2. 
Table 2. Comparison of functional recovery of knee joint between group A and group B after operation for complex tibial plateau fractures.

\begin{tabular}{ccccccc}
\hline Group & $\begin{array}{c}\text { Excellent } \\
\text { (case) }\end{array}$ & Good (case) & Fair (case) & Total cases & $\begin{array}{c}\text { Excellent and } \\
\text { good rate (\%) }\end{array}$ & $\begin{array}{c}\text { Excellent } \\
\text { rate }(\%)\end{array}$ \\
\hline $\begin{array}{c}\text { Control } \\
\text { group }\end{array}$ & 5 & 7 & 2 & 14 & 85.71 & 35.71 \\
$\begin{array}{c}\text { Observation } \\
\text { group }\end{array}$ & 11 & 2 & 1 & 14 & 92.86 & 78.57 \\
$\chi^{2}$ & & & & & $\chi^{2}=0.373$ & $\chi^{2}=5.250$ \\
$P$ & & & & 0.54 & $<0.05$ \\
\hline
\end{tabular}
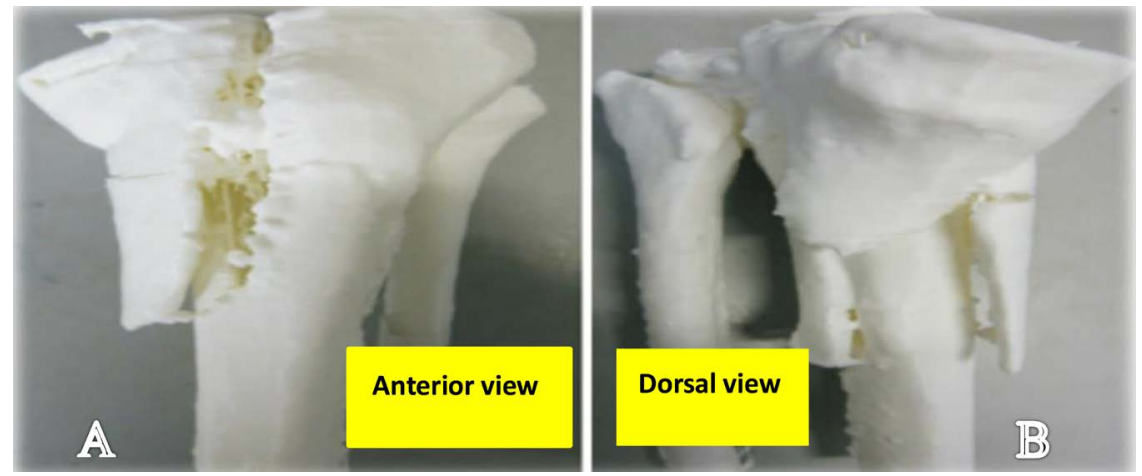

Figure 1. The Anterior view and Dorsal view of 3D printing model.

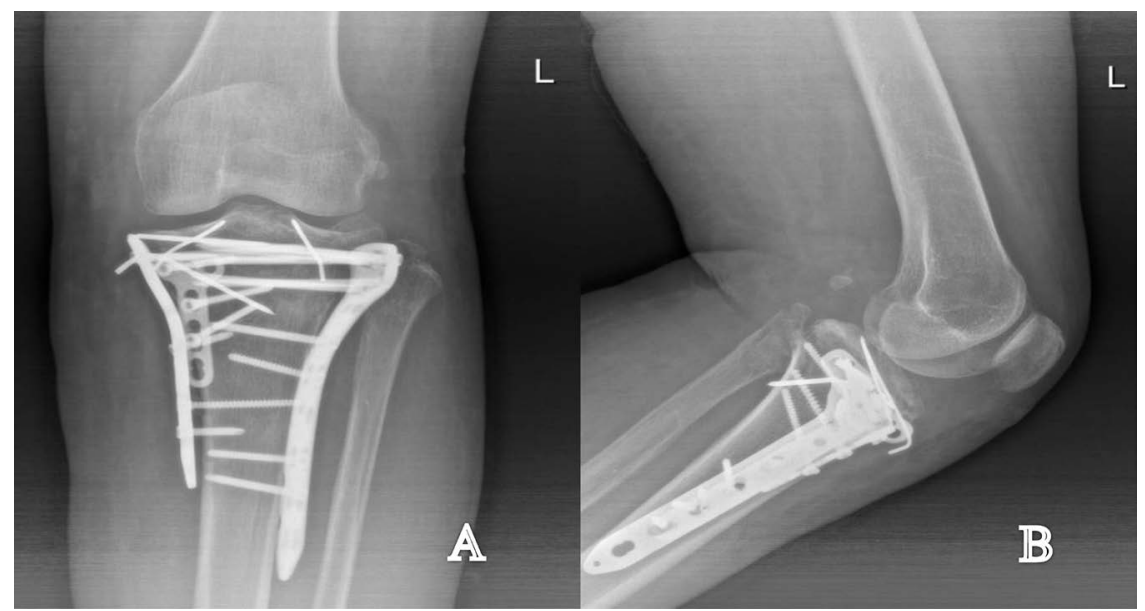

Figure 2. X-rays was reexamined one month after surgery.

\section{Discussion}

\subsection{The Characteristics of Tibial Plateau Fractures and the Commonly Used Treatment Methods in China and Abroad}

Tibial plateau fracture is a complex intra-articular fracture. Schatzker proposed in 1979 to classify tibial plateau fracture into six types [4], which has been used up to now. However, it was found in clinical practice that fractures combined with the posterior bone block of the tibial plateau were not well applicable to the Schatzker classification. Based on this, Luo Congfeng et al. [5] proposed a Three- 
column Classification of tibial plateau fractures based on CT scan results in 2012. The tibial plateau was divided into lateral column, medial column and posterior column. Cortical rupture was defined as column fracture, and the collapse of tibial plateau articular surface without cortical rupture was defined as zero column fracture. Three-column Classification can better guide orthopaedics to choose surgical approaches and internal fixation methods, so as to obtain better reduction and more stable fixation, which is conducive to the recovery of knee joint function, and significantly improve the therapeutic effect. In recent years, Zhang Yingze et al. [6] have proposed that the current classification cannot include the special type of tibial plateau fracture combined with fibular head fracture, so a new 6-type comprehensive classification method is proposed to make the surgical treatment of tibial plateau fracture more integrated. According to different types of fractures, the treatment methods are also different, and individualized surgical plans should be made according to different types of fractures. With the development of modern orthopaedics and the deepening of research on tibial plateau fractures, the concept of treatment is constantly updated, and the treatment methods are gradually improved and perfected. Limited incision, minimally invasive technique, indirect reduction by prying technique, application of external fixator and biological fixation are the future development directions of tibial plateau fracture treatment [7].

\subsection{Advantages of 3D Printing Technique over Other Methods}

\subsubsection{Preoperative Planning of Sham Operated}

Preoperative design of complex tibial plateau fracture is formulated by using $3 \mathrm{D}$ printing technique to simulate the operation of virtually reduction and internal fixation of complex tibial plateau fracture before operation [8]. In this way the fracture can be accurately and effectively reduced and fixed during operation, the operative blood loss can be reduced, the success rate of one-time placement of implants and devices can be increased, and the accuracy of surgery can be improved, it is considered as a relatively simple and practical clinical tool [9].

\subsubsection{Accuracy of Intraoperative Navigation}

The application of navigation template for $3 \mathrm{D}$ printing technique can help to accurately insert the bone fracture plate and screw, reduce the penetration rate and direction error rate of screw placement [10] [11], and avoid iatrogenic osteoporosis and internal fixation loosening caused by repeated screw placement.

\subsubsection{Clinical Teaching and Surgical Training}

Through the intuitive understanding of 3D printing model, medical students and young doctors can not only diagnose and classify accurately, but also improve the understanding of complex fractures, which is conducive to the growth of young doctors and shorten the learning curve [12] [13].

\subsubsection{Facilitate Doctor-Patient Communication and Understanding}

The application of $3 \mathrm{D}$ printing technique can effectively promote the doctor-pa- 
tient communication, in conversation with the patient, using $3 \mathrm{D}$ printing technique to make the model vividly and describe the disease condition to patients and their families and inform them of the process during the operation, so that patients can truly feel "clear" treatment, effectively alleviate the tension between patients, with significant clinical benefits [14]. Therefore, it is an effective tool to help doctors and patients communicate [15].

\subsection{Problems in the Promotion and Application of 3D Printing Technique}

Although preliminary studies have shown that 3D printing technique can improve the therapeutic effect of fracture surgery and functional recovery after surgery. However, in clinic, there are still many difficulties in making 3D printing model and applying and popularizing virtually reduction technology [16]. 1) Increase the total cost of surgery, increase the financial burden of patients seeking medical treatment. 2) Printing of individualized prostheses is still in the scientific research stage, and commercial promotion is still limited by the application of biomaterials, whether it can adapt to long-term high-intensity use is still unknown. 3) The relevant clinical guidelines and laws and regulations are faultiness: 3D printing technique involves many fields such as intellectual property rights, human ethics, dangerous goods manufacturing and so on. At present, there is no clear related policies and laws to complement with it. 4) Low timeliness: From the establishment of imaging data to the printing of physical models and the manufacture of individualized prostheses and internal plants, the whole process takes hours to days, so it is difficult to be used in emergency surgery.

\section{Prospect of 3D Printing for Tibial Plateau Fractures}

The development and application of 3D printing technique has indeed improved the therapeutic effect and functional recovery of traditional tibial plateau fracture surgery, and made individualized and precise medical treatment possible. However, how to further control the cost, research and development of biomaterials and gradually popularize the application are still worth considering. With the rapid development of digital medicine and the deepening of research, the application of computer software-assisted surgery, surgical robots, complex tibial plateau fractures and other complex trauma diagnosis and treatment will be more rapid and effective.

\section{Conflicts of Interest}

The authors declare no conflicts of interest regarding the publication of this paper.

\section{References}

[1] Gardner, M.J. and Schmidt, A.H. (2014) Tibial Plateau Fractures. Journal of Knee Surgery, 27, 3-4. https://doi.org/10.1055/s-0033-1363854 
[2] Wang, L. and Dai, K.R. (2014) Individualized Treatment of Orthopaedics and 3D Printing Technology. Journal of Medical Biomechanics, 29, 193-199.

[3] Cho, W.S., Park, S.S., Kim, D.H., et al. (2000) The Reliability of HSS Knee Rating System.

[4] Schatzker, J., Mcbroom, R., Bruce, D., et al. (1979) The Tibial Plateau Fracture. The Toronto experience 1968-1975. Clinical Orthopaedics, 138, 94-104.

[5] Luo, C.F., Hu, C.F., Gao, H., et al. (2009) Three-Column Classification for Tibial Plateau Fractures. Chinese Journal of Orthopaedic Trauma, 11, 201-205.

[6] Chang, Z.L., Chang, H.R., Zhang, Y.Z., et al. (2018) Preliminary Study on Comprehensive Classification of Tibial Platform Fractures. Journal of Hebei Medical University, 39, 1354-1355.

[7] Editorial Committee of Chin J Orthop Trauma (2015) Expert Consensus on Diagnosis and Treatment of Tibial Plateau Fractures. Chinese Journal of Orthopaedic Trauma, 17, 1671-7600.

[8] Meng, G.L., Liu, J., Hu, Y.Y., et al. (2011) Rapid Prototyping Used in the Operation Design for Complex Fractures of Tibial Plateau. Chinese Journal of Orthopaedic Trauma, 13, 1135-1138.

[9] Dong, L.L., Guo, P.M., Zuo, Q., et al. (2016) Application of 3D Printed Fracture Model in Schatzker Classification of Tibial Platform Fractures. Chinese Journal of Bone and Joint Injury, 31, 860-861.

[10] Lu, S., Zhang, Y.Z., Wang, Z., et al. (2012) Accuracy and Efficacy of Thoracic Pedicle Screws in Scoliosis with Patient-Specific Drill Template. Medical \& Biological Engineering \& Computing, 7, 751-758. https://doi.org/10.1007/s11517-012-0900-1

[11] Kawaguchi, Y., Nakano, M., Yasuda, T., et al. (2012) Development of a New Technique for Pedicle Screw and Magerl Screw Insertion Using a 3-Dimensional Image Guide. Spine, 23, 1983-1988.

[12] Sodian, R., Schmauss, D., Schmitz, C., et al. (2009) 3-Dimensianal Printing of Medels to Create Custom-Made Devices for Coil Embolization of an Anastomotic Leak after Aortic Arch Replacement. The Annals of Thoracic Surgery, 88, 974-978. https://doi.org/10.1016/j.athoracsur.2009.03.014

[13] Kim, G.B., Lee, S., Kim, H., et al. (2016) Three-Dimensional Printing: Basic Principles and Applications in Medicine and Radiology. Korean Journal of Radiology, 17, 182-197.

[14] Yi, C.R., Luo, J.S., Wang, W.J., et al. (2015) Progress of 3D Printing Technology in Individualized Treatment of Bone Tissue Repair. Medical Science Journal of Central South China, 43, 330-333.

[15] Yang, L., Shang, X.W., Fan, J.N., et al. (2016) Application of 3D Printing in the Surgical Planning of Trimalleolar Fracture and Doctor-Patient Communication. BioMed Research International, 2016, 2482086.

[16] Huo, L.F. and Ni, H.J. (2015) Application and Prospects of Digital Orthopedics: More Precise, Individual and Intuitional Outlook. Chinese Journal of Tissue Engineering Research, 19, 1457-1462. 